\title{
RELACIÓN ENTRE EL CURRICULUM EN CARRERAS DE FORMACIÓN DE PROFESIONALES DE EDUCACIÓN FÍSICA Y EL MANIFIESTO MUNDIAL DE LA EDUCACIÓN FÍSICA
}

\section{RELATIONSHIP BETWEEN THE CAREER TRAINING CURRICULUM IN PHYSICAL EDUCATION PROFESSIONALS AND THE WORLD MANIFESTO OF PHYSICAL EDUCATION}

\author{
Portes Junior, Moacyr ${ }^{1}$ \& Vargas, Ángelo Luis de Souza ${ }^{2}$ \\ ${ }^{1}$ Carrera de Pedagogía en Educación Física de la Universidad Autónoma de Chile, sede Talca. \\ 2 Universidade Federal do Rio de Janeiro y de la Universidade Estácio de Sá, Rio de Janeiro, Brasil.
}

PORTES J. M. \& VARGAS A. L. S. Relación entre el Curriculum en Carreras de Formación de Profesionales de Educación Física y el Manifiesto Mundial de la Educación Física. Mot.Hum. 13(2): 68-73; 2012.

\section{RESUMEN}

El desarrollo del área de la Educación Física escolar chilena está creciendo en importancia, en calidad y en exigencias, considerando la realidad socio cultural de Chile. Existen referentes nacionales e internacionales para la Educación Física, que deben ser considerados en la implantación de un plan de estudios para formación de docentes para esta área. Este estudio busca verificar si las universidades que imparten la carrera de Pedagogía en Educación Física en la ciudad de Chillán, tienen sus planes de estudios, indicados por las mallas de las carreras, coherentes con el referencial mundial de la Educación Física, que es el Manifiesto Mundial de la Educación Física, FIEP. La metodología estuvo basada en un estudio exploratorio, documental; análisis de los datos a partir de estadística descriptiva. La muestra son las tres carreras impartidas en la ciudad de Chillán, por tres universidades: dos particulares y una pública. Los resultados: Institución "A", entrega asignaturas que abarcan $100 \%$ de los veinte tres capítulos del Manifiesto Mundial de la Educación Física; Institución "B", 73,91\%; Institución "C", 82,60\%. Se puede concluir que, en lo que se refiere a aspectos de fondo, de formación específica de la Educación Física, las carreras en la ciudad de Chillán, están fuertemente basadas, necesitando ajustes en su plan de estudios, en aspectos generales de formación profesional y de la comprensión de la epistemología de la Educación Física, como también, de las relaciones político-administrativas que el futuro profesional deberá tener, para desarrollar su práctica profesional, tanto en la escuela, cuanto en situaciones sociales, extra escolares, de la Educación Física.

Palabras claves: Manifiesto Mundial de Educación Física, Planes de Estudio, Pedagogía en Educación Física.

\begin{abstract}
The development of the Chilean scholar Physical Education's area is growing in importance, in quality and exigencies, considering the cultural and social reality of Chile. Exist national and international references for the Physical Education that must be considered in the implantation of a curriculum to formation of teachers to this area. This study looks for to verify if the universities that distribute the graduation in Physical Education Pedagogy in the city of Chillan, have the curriculum, indicated by the curricular structure of the courses, coherent with world referential of the Physical Education, that is the World Manifest of the Physical Education, FIEP. The methodology was based on an exploratory, documentary study; analysis of the dates from descriptive statistic. The sample is the three courses distributed in the city of Chillan: two privates and one public. The results: Institution "A", gives disciplines that include 100\% of the twenty three chapters of the World Manifest of the Physical Education; Institution "B", 73.91\%; Institution "C", 82.60\%. It is possible to be concluded that, in which talk about bottom aspects, of the specific formation of the Physical Education, the courses in the city of Chillán, are strongly based, needing adjustments in the curriculum, in general aspects of professional formation and of the understanding of the epistemology of the Physical Education, like also, of the political-administrative relations that the future professional must have, to develop in the professional practice, as much in the school, whatever in social situations, extra-scholar, of the Physical Education.
\end{abstract}

Key Words: World Manifest of Physical Education, Curricular Structure of the Courses, Physical Education Pedagogy. 


\section{INTRODUCCIÓN}

Implementar una propuesta pedagógica, además de las dificultades inherentes al proceso, revela el empeño en participar de una tarea compleja, tanto cuando se considera la variable epistemológica, cuanto a adecuación de esta propuesta a la realidad social existente (1). En el área de Educación Física esa realidad posee las mismas dificultades y por veces, mayores en se tratando de las características específicas del área y de las necesidades que as sociedad occidental está enfrentando en relación a la salud, al sedentarismo, al aumento de las enfermedades crónico degenerativas, el aumento de los índices de obesidad y sobrepeso $(2,3,4)$ y a la tendencia del ser humano de la pérdida de la capacidad del movimiento intencional (5), en busca de la mantención de la salud (6), que es el fin de la Educación Física.

Bajo esta perspectiva, una Carrera de Enseñanza Superior en Educación Física, debe estar establecida de tal manera que pueda entregar a los estudiantes, los conocimientos necesarios para su actuación profesional en la realidad social en la cual está inserta.

Una propuesta pedagógica en Carreras Superiores en Educación Física dentro de esta perspectiva, debe estar centrada en el movimiento y en el desarrollo del repertorio motor de los alumnos para que puedan maximizar las posibilidades de participación en actividad que puedan auxiliar a la mantención de la salud física o de la recuperación de esta posibilidad, transformando la Educación Física en una de las herramientas más importante en el proceso de cambios sociales en relación al comportamiento y a la educación para la salud $(7,8,9)$.

Este compromiso de la formación en Educación Física con la Educación crece en la medida en que se reconoce la necesidad del movimiento, tanto para los objetivos anteriormente mencionados, cuanto para los objetivos relacionados al desarrollo cognitivo, afectivo y social de los estudiantes (10).

Históricamente la formación en Educación Física ha pasado por dificultades relacionadas a esa relación con la Educación integral, cuando se percibía un direccionamiento de las clases en las escuelas, en la búsqueda del rendimiento físico como objetivo de las clases; falta de una mirada que fuera capaz de establecer la asignatura dentro de las posibilidades inter y transdisciplinarias del proceso educacional; falta de una percepción de necesidad de planificación de la acción educativa y de estrategias que consideraran la realidad y las experiencias de los alumnos; la vinculación con el entendimiento de que la Educación Física era solamente práctica deportiva o momento de recreación para compensar el esfuerzo exigido por las demás asignaturas de la malla curricular de la Enseñanza Básica y Media $(11,12,13)$.

Todos estos factores relacionados a la Educación Física escolar, tuvieron impacto en el establecimiento de los planes de estudio de las carreras formadoras de profesionales del área. La idea central de una propuesta de Carrera de formación en Educación Física está en el compromiso de entregar una capacitación que demuestre posibilidades de cambiar la realidad histórica y ajustar la realidad actual, a las necesidades de la sociedad, a que la propuesta y los futuros profesionales han de ser entregados.

El proceso de elaboración de la propuesta consolida la dirección de integralidad de la Educación Física con la realidad educativa y a los modelos pedagógicos escolares. Además de definir claramente su función social dentro de las políticas públicas relacionadas a la salud, reconociendo el ser humano que debe participar de ella y de los medios a utilizar para alcanzar los objetivos de estos, buscando la importancia de la actividad física para el desarrollo biopsicosocial del estudiante (14).

Considerando los aspectos pedagógicos, una propuesta de carrera de formación debe estar establecida con fines de capacitar los profesionales que han de trabajar en el área a planificar, desarrollar y evaluar los procedimientos pedagógicos de la motricidad humana en sus diferentes contextos (15), consumando la Educación Física en su función dentro de las Instituciones educacionales, donde estén presentes los principios de crecimiento y desarrollo del ser humano y su relación con las cuestiones de enseñanza y aprendizaje reconociendo el ser humano como un ser que se movimiento y que necesita de este movimiento, intencionalmente, para la mantención de su salud $(16,17)$. 
En la lógica de estas ideas la propuesta debe avanzar en el sentido del alcance de la ciudadanía, donde el estudiante pueda en su práctica profesional futura, estimular sus alumnos a participar de programas que han de intentar lograr su realización y satisfacción, para que esta afectividad creada, redunde en un comportamiento continuo y consciente para el comprometimiento en responder los "porqués" de hacerse y "para que" hacerse, de manera contextualizada y centrada en las relaciones humanas de la construcción colectiva de una sociedad que se preocupa con la mantención y la búsqueda de la salud. Esto no significa una Educación Física moldada en los patrones de estética, de belleza o perfección y si de una consciencia corporal adecuada en los valores de igualdades de posibilidades de realización, de integración y de inclusión; no competitiva en el sentido de sobreponerse, pero competitiva en el sentido de la auto superación y cooperativa en el sentido de los valores de la socialización y sociabilización $(18,19)$.

La propuesta debe entregar sugerencias de vivencias que deben ser experimentadas por los estudiantes, privilegiando la participación del alumno en diversas rutinas prácticas, interactuando la teoría para la práctica consciente $(20,21,22)$.

También, entregar a los futuros docentes las informaciones relacionadas a los aspectos didácticos pedagógicos y de los momentos de evaluación del proceso, para que sea capaz de buscar la coherencia entre la realidad y su práctica docente, entregando a él (o ella) el camino del compromiso social de la Educación Física como productora de los beneficios pretendidos en los objetivos establecidos $(23,24,25)$.

Los aspectos didácticos pedagógicos deben proporcionar el conocimiento profesional necesario para buscar las formas de organización curricular para aprovechar el espacio y el tiempo disponibles para el trabajo; diferentes modos de desenvolvimiento del trabajo para la producción y participación colectiva, proporcionando al alumno las mejores condiciones para su desarrollo, en un espacio de construcción de acciones deseables y estimulantes para esto.

Es un desafío la construcción de competencias del profesional y de los estudiantes para la formación integral preconizada, articulada con las necesidades individuales y con modos de acción significativos $(26,27,28)$.

De los conocimientos a enseñar y de los conocimientos educacionales, las propuestas deben contemplar espacios, actividades, materiales y tiempos de actividades que permitan la transposición de los conocimientos prácticos para transformarlos en conocimientos teóricos para la educación para la salud y auto gestión del desarrollo personal (29).

Todo el hacer implica reflexión y comprensión del sentido, buscando los significados y razones involucradas en esta práctica, con diferentes contenidos y conocimientos para su aplicación personal, social, en la perspectiva didáctica, al mismo tiempo que garantiza su aplicación en el cotidiano del alumno (30). Que las manifestaciones de la cultura corporal puedan tener un enfoque didáctico metodológico de forma a garantizar que los alumnos reconozcan las posibilidades y avances en la búsqueda de los valores necesarios para las vivencias prácticas en la actividad física, para el cuidado de la salud, para las vivencias en situaciones de socialización, de actividades lúdicas, de carácter utilitario, comprendiendo los objetivos de esto.

Por fin, una propuesta de Carrera de Formación de futuros Profesionales de Educación Física, debe estar basada, también, en los principios que orientan la profesión en nivel mundial, y la referencia para estos es el Manifiesto Mundial de la Educación Física, que entrega los referentes del área.

El objetivo general de este estudio es verificar si las carreras de Pedagogía en Educación Física, establecidas en la ciudad de Chillán, tienen, en su malla curricular, asignaturas que indiquen la relación y la coherencia entre la propuesta formadora de futuros profesionales, con los referentes del área de Educación Física mundial, en lo que se refiere al Manifiesto Mundial de la Educación Física, de la Federación Internacional de Educación Física, FIEP.

Y como objetivos específicos están los de verificar si las mallas curriculares, establecidas en los Planes de Estudios de estas carreras, presentan asignaturas que se relacionan a los veinte y tres capítulos establecidos como referencia para la 
Educación Física mundial. También, cuanto de la formación entregada por las carreras, en indicadores cuantitativos, está establecida bajo los criterios internacionales de la Educación Física. Por fin, verificar si la formación entregada en las carreras es coherente con las exigencias internacionales para la intervención del profesional de Educación Física.

\section{MATERIAL Y MÉTODOS}

A partir de un estudio exploratorio, documental, analizar las mallas curriculares de las carreras de Pedagogía en Educación Física, impartidas en la ciudad de Chillán, Chile, buscando:

1. Qué relación las asignaturas presentadas en la malla curricular, tienen con los veinte tres capítulos del Manifiesto Mundial de la Educación Física, en lo que se refiere a los principios de cada capítulo y la relación de la asignatura, expresada en la descripción de la asignatura.

2. El análisis de los datos será a partir de análisis descriptiva - estadística descriptiva - de la relación de las asignaturas de la malla curricular y los veinte tres capítulos del Manifiesto Mundial de la Educación Física.

3. Estos datos han de ser referencia para establecer la relación entre la formación y su coherencia con las exigencias del área en el ámbito internacional, expresada en datos cuantitativos.

La población está en la Universidades de la ciudad de Chillán, Chile, que imparten Carreras de Pedagogía en Educación Física. La muestra son tres Instituciones y tres Carreras, respectivamente.

Fueron tomadas las mallas de las carreras indicadas y analizadas, a partir de las asignaturas presentadas en la malla, la relación de estas asignaturas con los XXIII capítulos del Manifiesto Mundial de la Educación Física, considerando los principios establecidos en cada uno de los capítulos y los temas indicados en las asignaturas presentadas en la malla.

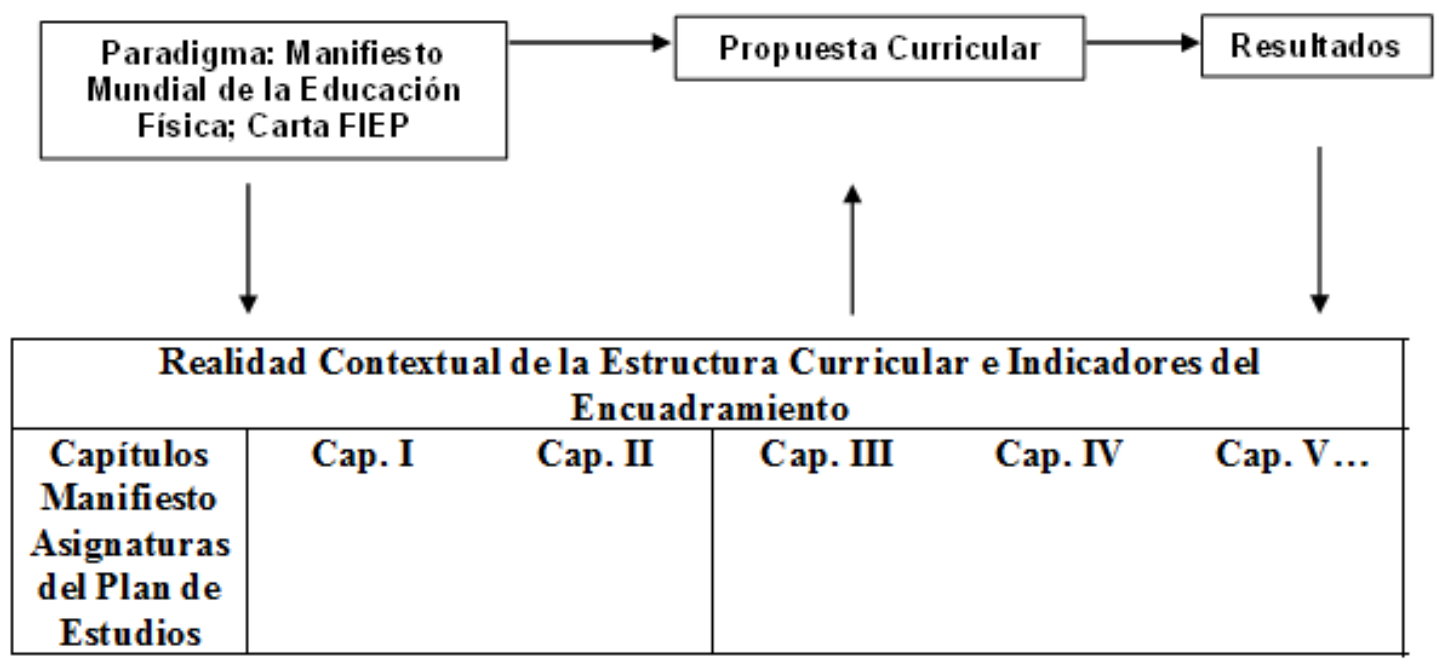

Figura 1: Esquema grafico de la metodología utilizada en el análisis.

\section{RESULTADOS}

A partir del estudio documental, exploratorio, se hizo la relación entre los veinte tres capítulos del Manifiesto Mundial de la Educación Física y las mallas de las carreras, buscando, en las mallas, asignaturas que en su contexto, entregaban contenidos y formación a los estudiantes, en los temas referentes al capítulo del Manifiesto. Se pudo verificar lo siguiente:

- La carrera de Pedagogía en Educación Física de la Institución "A", entrega asignaturas que abarcan $100 \%$ de los veinte tres capítulos del Manifiesto Mundial de la Educación Física.

- La carrera de Pedagogía en Educación Física de la Institución "B”, entrega 
PORTES J. M. \& VARGAS A. L. S. Relación entre el Curriculum en Carreras de Formación de Profesionales de Educación Física y el Manifiesto Mundial de la Educación Física. Mot.Hum. 13(2): 68-73; 2012.

asignaturas que abarcan $73,91 \%$ de los veinte tres capítulos del Manifiesto Mundial de la Educación Física, significando asignaturas que desarrollan contenidos para 17 de los capítulos. No se encontró relación de asignaturas con los Capítulos II, VII, XVII, XXI, XXII y XXIII.

- La carrera de Pedagogía en Educación Física de la Institución "C", entrega asignaturas que abarcan $82,60 \%$ de los veinte tres capítulos del Manifiesto Mundial de la Educación Física, significando asignaturas que desarrollan contenidos para 19 de los capítulos. No se encontró relación de asignaturas con los Capítulos XIV, XVIII, XXI y XXII.

\section{DISCUSION}

A partir de los resultados se pudo percibir que la Institución "B", adolece de la entrega de información en relación a los fundamentos epistemológicos de la Educación Física, las relaciones de la Educación Física para la salud, formación en proyectos que puedan buscar suplir necesidades emergentes en la Educación Física, tanto local, cuanto internacional, en lo que se refiere a países con carencias en esta área. También, las relaciones de la Educación Física con los medios externos, gubernamentales y específicos del área.

En la Institución "C", se verificó la carencia e asignaturas que entreguen formación en el área de metodologías de la Educación Física, para adaptaciones curriculares de acuerdo con realidades diferenciadas de materiales $y$ condiciones específicas; también, en lo que se refiere a comprensión ética para la paz, a partir de la postura de un educador ético. Y por fin, se verificaron ausencias de asignaturas que discutan aspectos correspondientes a las relaciones de la Educación Física con los medios externos, gubernamentales y específicos del área.

Se puede concluir entonces, que, en lo que se refiere a aspectos de fondo, de formación específica de la Educación Física, las carreras en la ciudad de Chillán, están fuertemente basadas, necesitando ajustes en su plan de estudios, en aspectos generales de formación profesional y de la comprensión de la epistemología de la
Educación Física, como también, de las relaciones político-administrativas que el futuro profesional deberá tener, para desarrollar su práctica profesional, tanto en la escuela, cuanto en situaciones sociales, extra escolares, de la Educación Física.

Además, indica que las carreras en la ciudad de Chillán, confirman por su Plan de Estudios, los lazos que están siendo firmados con la FIEP Federación Internacional de Educación Física, que establecen las directrices, tanto de la formación entregada, cuanto en lo que se refiere a eventos académicos de complementación curricular y de secuenciación de los estudios (especializaciones, congresos, post grados), entregados por las Instituciones de la ciudad. Esto también indica que Chile mantiene su fuerte vínculo histórico con las bases de la Educación Física.

\section{REFERENCIAS BIBLIOGRÁFICAS}

1. World Health Organization. Diet nutrition and prevention diseases. Report of Joint WHO/FAO Expert Consultation. Geneva: World Health Organization; 2002. (WHO Technical Report Series, 916).

2. Pereira, A.; Guedes, A.D.; Verreschi, I.T.N.; Santos, R.D.; Martinez, T.L.R, La obesidad y su asociación con los demás factores de riesgo cardiovascular en escolares de Itapetininga, Brasil, Arq Bras Cardiol. 2010; 93(3): 253-260.

3. Trujillo-Hernandez, B.; Vasquez, C.; Almaza-Silva, J.R.; Jaramillo-Virgen, M.E.; Mellin-Landa, T.E.; ValleFigueroa, O.B.; Pérez-Ayala, R.; Millán-Guerrero, R.O.; Prieto-Dìaz-Chávez, E.; Newton-Sánchez, O. Frecuencia y factores de riesgo asociados a sobrepeso y obesidad en universitarios de Colima, México, Rev. Salud Pública. 2010; 12(2):197-207.

4. Abbes, P.T.; Lavrador, M.S.F.; Escrivão, M.A.M.S.; Taddei, J.A. de A.C., Sedentarismo e variáveis clínicometabólicas associadas à obesidade em adolescentes, Rev. Nutr.2011; 24(4):529-538.

5. Ferreira, M.S.; Castiel, L.D; Cardoso, M.H.C. de A.; Atividade física na perspectiva da Nova Promoção da Saúde: contradições de um programa institucional, Ciênc. saúde coletiva. 2011; 16 (supl.1); 865-872.

6. Esper Di Cesare, P., La Educación Física Escolar como Vía de Educación para la Salud, Dirección de Educación Física de la Provincia de Buenos Aires, Disponible en: http://sportsalut.com.ar/articulos/act_fis_salud/20.pdf.2012.

7. Choque Larrauri, R., Comunicación y Educación para la Promoción de la Salud, MINISTERIO DE SALUD, (2000): 
PORTES J. M. \& VARGAS A. L. S. Relación entre el Curriculum en Carreras de Formación de Profesionales de Educación Física y el Manifiesto Mundial de la Educación Física. Mot.Hum. 13(2): 68-73; 2012.

"Lineamientos y Estrategias para la Gestión Local de la Comunicación Educativa”, Editora Perú.

8. Kain, J.; Concha, F.; Salazar, G.; Leyton, B.; Rodríguez, Medel P.; Ceballos, X.; Vio, F. Prevención de obesidad en preescolares y escolares de escuelas Municipales de una Comuna de Santiago de Chile: proyecto piloto 2006, ALAN 2009; 59: 139-46.

9. Hallal, P.C.; Knuth, A.G.; Cruz, D.K.A.; Mendes, M.I.; Malta, D.C., Prática de atividade física em adolescentes brasileiros, Ciênc. saúde coletiva. 2010; 15 (supl.2): 30353042 .

10. Bracht, V., A Constituição das Teorias Pedagógicas da Educação Física, Cadernos Cedes, ano XIX, $n^{\circ} 48$, Agosto/99.

11. Moulin, A.F.V.; Pereira, M. de M., Educação Física: Fundamentos para a Intervenção do Profissional Provisionado, Conselho Federal de Educação Física, 2006.

12. Palafox, G.H.M.; Terra, D.V.; Pirolo, A.L.; Amaral, G.A., Educação Física Escolar: conceito e fundamentos filosófico-pedagógicos para o pctp/ef, pctp/ef: Planejamento Coletivo do Trabalho Pedagógico. Estratégia de Intervenção crítica utilizada no processo de formação continuada de professores/as de Educação Física das redes públicas de ensino, em desenvolvimento desde 1993, Universidade Federal de Uberlandia, 2002.

13. Lucea, J.D., El Curriculum de la Educación Física en la Reforma Educatriva, 2ª ed, Barcelona: INDE, 1995.

14. Conselho Federal de Educação Física, Intervenção do Profissional de Educação Física, Rio de Janeiro, 2002.

15.Mattos, A.D.; Santos, J.F.S.; Cardoso, P.R.; Antonio, T., Atividade Física na Sociedade Tecnológica, http://www.efdeportes.com/ Revista Digital - Buenos Aires Año 10 - N 94 - Marzo de 2006.

16. Souza, E.A.de; Barbosa Filho, V.C.; Nogueira, J.A.D.; Azevedo Júnior, M.R.de, Atividade física e alimentação saudável em escolares brasileiros: revisão de programas de intervenção. Cad Saude Publica. 2011; 27(8): 1459-1471

17. Pérez Ordás, R.; Hernández Hernández, E.; García Sánchez, I., Relación entre el nivel de habilidad motriz y la ansiedad percibida antes y después de una práctica de escalada en niños de Educación Primaria, Retos. Nuevas tendencias en Educación Física, Deporte y Recreación, 2011;19: 25-29.

18. Baena Extremera, A.; Granero Gallegos, A. Las actividades físicas en la naturaleza en el currículum actual: contribución a la educación para la ciudadanía y los derechos humanos, Retos: nuevas tendencias en educación física, deporte y recreación. 2008; 14: 48-53.

19. Villarruel Fuentes, M., La Práctica Educativa del Maestro Mediador, Revista Iberoamericana de Educación, n. ${ }^{\circ} 50 / 3-10$ de septiembre de 2009.
20. Falkenbach, A.P., Educação física na educação infantil: o futuro professor na relação com as crianças, Rev. bras. ciênc. Mov. 2006; 14(1): 21-28.

21. Moreno, J.A.; Martínez, A., Importancia de la teoría de la autodeterminación en la práctica físico-deportiva: fundamentos e implicaciones prácticas, Universidad de Murcia, Espinardo (Murcia), España, Cuadernos de Psicología del Deporte. 2006; 6(2), 39-54.

22. Sánchez Bañuelos, F., Bases para una Didáctica de la Educación Física y el Deporte, Madrid: Gymnos, 1992.

23. Chile, Marco Curricular de la Educación Básica, Ministerio de Educación, Gobierno de Chile, 2002.

24. Chile. Programa de Estudio, Primer Año Básico, Educación Física, Ministerio de Educación, Gobierno de Chile, 2002.

25. Chile, Actualización Curricular 2009, Ministerio de Educación, Gobierno de Chile, 2009.

26.Sánchez Bañuelos, F., Didáctica de la Educación Física para Primaria, España, Pearson Educación, 2002.

27. Carlan, P.; Domingues, S.C.; Kunz, E. Didática da Educação Física Brasileira: uma compreensão da produção científica. Revista Pensar a Pratica. 2009; 12 (3): 1-11.

28. Silva, R.S.; Silva, I.; Silva, R.A.; Souza, L.; Tomasi, E., Atividade física e qualidade de vida, Ciência \& Saúde Coletiva, 2010; 15(1):115-120.

29. Pinto, J.R.; Fernandez Filho, J.; DANTAS, E.M.H., Aptidão: Qual? Para Que?, Rev. Bras. De Cinenat. Desemp. 2000; 2(1), 80-88.

30. Federación Internacional de Educación Física, Manifiesto Mundial de la Educación Física, disponible en: www.fiepchile.cl.

Dirigir Correspondencia a:

Moacyr Portes

portes.junior@yahoo.com.br

5 Poniente 1670, Talca. Chile.

RECIBIDO: 16-08-2012

ACEPTADO: 7-11-2012 